ISSN 0001-6012/2014/56/2/65-70 Acta Médica Costarricense, ( 2014 Colegio de Médicos y Cirujanos de Costa Rica

\title{
Original
}

\section{Recolección de células madre en sangre periférica mediante aféresis de grandes volúmenes}

\section{(Peripheral blood stem cell collection by apheresis)}

\author{
Greivin Zumbado-Salas ${ }^{1}$, Miguel Rodríguez-Pineda², Xinia Rojas-Camacho33, Carlos Rojas-Solano ${ }^{3}$ y \\ Hever Herrera-Álvarez ${ }^{3}$
}

\section{Resumen}

Antecedentes: evaluar el rendimiento y calidad de las recolecciones de células madre en sangre periférica realizadas en el Hospital México entre junio del 2010 hasta Junio del 2012, dado que existe una dosis mínima de células CD34+ (células progenitoras hematopoyéticas) que definen la posibilidad de éxito del injerto en el trasplante de células madre.

Métodos: estudio retrospectivo utilizando los registros de los pacientes y los procedimientos de recolecciones de células madre por aféresis efectuados en el servicio de hematología Hospital México. Para evaluar el rendimiento y calidad de las recolecciones y el efecto de estas en los tiempos de recuperación de los parámetros hematológicos de los pacientes trasplantados entre Junio del 2010 y Junio del 2012.

Resultados: se realizaron 52 procedimientos de aféresis a 26 pacientes (46\% Linfoma Hodgkin, 27\% Linfoma no Hodgkin, 27\% mieloma múltiple). Se procesaron en promedio un total de 8 volemias por paciente con una recolección media de $8.48 \times 10^{8}$ células mononucleares $/ \mathrm{Kg}$ recolectadas, de las cuales en promedio $3.06 \times 10^{6} / \mathrm{kg}$ corresponde a células $\mathrm{CD} 34^{+}$. El tiempo promedio de recuperación para los parámetros hematológicos por paciente fue de 15.05 días para leucocitos y 17.15 días para plaquetas. Se observó una correlación inversa entre el número de células CD34+ infundidas y el tiempo de recuperación de las plaquetas, no así para los leucocitos.

Conclusiones: los recuentos obtenidos para las recolecciones estuvieron dentro de los valores descritos en otras publicaciones, lo mismo que los tiempos de recuperación de los parámetros hematológicos post infusión, por lo que el protocolo utilizado ha mostrado ser exitoso. Solo el recuento de leucocitos pre recolección mostró ser de utilidad para predecir una buena recolección, además el recuento de células $\mathrm{CD} 34^{+}$infundidas al paciente es el mejor parámetro para predecir una buena implantación del injerto.

Descriptores: células madre, células $\mathrm{CD} 34^{+}$, aféresis, recolecciones.

Trabajo realizado en el Banco de Sangre Hospital México, Caja Costarricense de Seguro Social

Afiliación de los autores: 'Centro de Trasplante y Cirugía Hepatobiliar, Hospital México, Facultad de Medicina Universidad de Costa Rica. 2Servicio de Hematología Hospital Calderón Guardia, Facultad de Microbiología Universidad de Costa Rica. ${ }^{3}$ Banco de Sangre Hospital México

凶rojasxe@gmail.com

\section{Abstract}

Background: To evaluate performance and quality of peripheral blood stem cell collections performed at the México Hospital from June 2010 to June 2012, since there is an established minimum dose of $\mathrm{CD} 34^{+}$cells that determines the probability of success of engraftment.

Methods: A retrospective study using patient records and data obtained from peripheral blood stem cell apheresis procedures carried out at the Haematology Unit of the México Hospital. This information was used to calculate performance and quality values of collections, and the effect of these on the recovery time of haematological parameters in patients transplanted between June 2010 and June 2012. 
Results: A total of 52 peripheral blood stem cell apheresis procedures were performed to 26 patients (Hodgkin lymphoma 46\%, non-Hodgkin lymphoma and multiple myeloma 27\%). An average of 8 blood volumes per patient was processed, the median collection was $8.48 \times 10^{8}$ mononuclear cells $/ \mathrm{Kg}$, of which an average of $3.06 \times 10^{6} / \mathrm{Kg}$ were $\mathrm{CD} 34^{+}$cells. The average recovery time of haematological parameters after transplant was 15.05 days for leukocytes and 17.15 days for platelets. An inverse correlation was observed between CD34+ infused cell count and time for platelet count recovery, but not for leukocyte count recovery.

Conclusions: Cell counts obtained from apheresis collections and recovery time of haematological parameters after transplant were consistent with results described by the medical literature, showing that the protocol followed has been successful. Only leukocyte count previous to apheresis has proved to be useful to predict a quality collection, and CD34+ infused cell count is the best parameter to predict a successful engraftment.

Keywords: stem cells, CD34+ cells, apheresis, collection.

Fecha recibido: 20 de enero de 2014

Fecha aceptado: 6 de marzo de 2014

El uso de altas dosis de quimioterapia, seguidas de un trasplante autólogo de precursores hematopoyéticos de sangre periférica, se ha convertido en un tratamiento de gran importancia para muchas malignidades hematológicas como mieloma múltiple, linfoma Hodgkin, linfoma no Hodgkin. ${ }^{1-4}$

Hasta no hace demasiado tiempo los precursores hematopoyéticos se obtenían de médula ósea, pero debido a que este procedimiento resulta más complicado, por la necesidad de ser practicado en quirófano, bajo los efectos de la anestesia y los riesgos por el trauma que involucran las múltiples punciones, esta fuente de recolección ha sido desplazada por células madre obtenidas de sangre periférica (PBSC por sus siglas en inglés). ${ }^{5,6}$ En los últimos años, el uso de PBSC para el trasplante autólogo y alogénico se ha incrementado significativamente. Según datos del Centro de Investigación Internacional de Trasplante de Sangre y Médula (CIBMTR), más del 95\% de los trasplantes autólogos de células madre (ASCTs) y más del 70\% de los trasplantes alogénicos se realizan con PBSC (movilizadas). Las ventajas de utilizar PBSC sobre el uso de células médula ósea son: tiempo de injerto más corto, menor necesidad de transfusiones, menor estancia hospitalaria, facilidad de recolección de las células, y restauración rápida del sistema inmunológico.,,6,7

La recolección de los precursores hematopoyéticos se lleva a cabo posterior a estimulación de la médula ósea para que las células progenitoras (CD34+) migren a sangre, facilitando su recolección de la circulación periférica a través de la realización del procedimiento de aféresis. Este procedimiento se conoce como movilización. ${ }^{2}$ Tradicionalmente, la movilización de PBSC para los trasplantes autólogos ha sido realizada utilizando solamente citoquinas (factores de crecimiento), o en combinación con quimioterapia. Sin embargo, los resultados han demostrado que existen grupos de pacientes incapaces de alcanzar la mínima dosis de PBSC requerida durante su primera ronda de movilización celular, y precisan una segunda ronda utilizando regímenes de salvamento. 6,8

Actualmente se hace cada vez más necesario identificar de forma precisa los pacientes con mayor riesgo de ser "pobres movilizadores", en los cuales no se logra recolectar la cantidad mínima necesaria de células CD34 $/ \mathrm{kg}\left(\geq 2 \times 10^{6} / \mathrm{Kg}\right)$ en el primer ciclo de movilización. Factores que favorecen una buena movilización son: diagnóstico de mieloma múltiple, movilización con quimioterapia, dosis de factor de crecimiento y conteos celulares adecuados antes de la recolección (leucocitos, CD34+ $>20 / \mu \mathrm{L}$ y plaquetas). Por el contrario, la edad avanzada, el sexo femenino, el diagnóstico de linfoma no Hodgkin, los tratamientos prolongados con quimioterapia y la historia de radioterapia, son factores predictores de una mala movilización. ${ }^{1,9}$ Otros factores determinantes en los rendimientos de las recolecciones de precursores hematopoyéticos en sangre periférica, son los ligados directamente al procedimiento: el acceso vascular, el flujo sanguíneo, el volumen total procesado, la experiencia del operador y las especificaciones del equipo de aféresis utilizado. ${ }^{1,2,10-12}$

En general, para un trasplante autólogo se considera una recolección exitosa cuando el conteo de células $\mathrm{CD}_{3} 4^{+} /$es de al menos 2 × $10^{6}$ células/Kg de peso, dosis suficiente para lograr el éxito del injerto, pero idealmente se prefieren recuentos mayores, ya que aceleran el injerto leucocitario y plaquetario y reducen las transfusiones y la hospitalización. En muchos casos este conteo no es alcanzado en un solo procedimiento, por lo que suele necesitarse al menos 2 recolecciones. ${ }^{1,2,7,11}$

El hecho de que exista una dosis mínima de células progenitoras que indiquen la posibilidad de éxito del injerto, y que esta dependa de múltiples factores que deben ser monitoreados antes y durante las recolecciones de precursores hematopoyéticos, son las razones que motivan este trabajo, para analizar el rendimiento y la calidad de las recolecciones realizadas a los pacientes del Servicio de Hematología del Hospital México.

\section{Métodos}

Estudio retrospectivo descriptivo, utilizando los registros de los procedimientos de aféresis realizados por el Banco de Sangre del Hospital México, las bases de datos del laboratorio Clínico 
del Hospital, y los expedientes de los pacientes como fuentes de información.

Se analizaron todas las recolecciones de células madre en sangre periférica efectuadas entre junio de 2010 hasta junio de 2012, a pacientes del Servicio de Hematología del Hospital México, con el fin de realizar trasplantes autólogos de médula ósea.

Durante este periodo se realizaron 52 recolecciones a 26 pacientes con padecimientos malignos hematológicos.

Movilización y acceso vascular: en todos los pacientes la movilización se realizó con G-CSF $5 \mu \mathrm{g} / \mathrm{kg} /$ día subcutáneo, durante 5 días previos a la recolección; las recolecciones se llevaron a cabo los días 6 y 7 . El día previo a la recolección con el paciente ya internado, se le colocó el acceso vascular, el cual fue en todos los casos un catéter central, casi siempre femoral.

Recolección de células progenitoras hematopoyéticas en sangre periférica: todas las recolecciones se realizaron en la Unidad de Trasplante de Células Madre Hematopoyéticas del Hospital México, en condiciones estrictamente controladas por personal entrenado para estos fines, y bajo monitoreo continuo de los signos vitales del donante. En todos los casos el donante se encontraba consciente y orientado, sin ningún tipo de sedación. Las recolecciones se realizaron con el equipo COBE Spectra (Caridian BTC, Denver, CO, USA), con el descartable para leucaferesis en el programa para recolección de células mononucleares. Se utilizó ACD como anticoagulante con 1000 unidades de heparina para trabajar a una proporción de 24:1, el hematocrito de recolección se estableció mediante el colorgrama entre el $1 \%$ y el $2 \%$. El objetivo era procesar al menos 3 volemias por procedimiento.

Parámetros hematológicos y de calidad: en los procedimientos se recopilaron los datos necesarios para realizar la recolección: peso, talla, volemia, conteo de leucocitos antes del procedimiento y hematocrito. Al final se cronometró el tiempo del procedimiento, se tomaron muestras de la recolección para recuento de leucocitos, células mononucleares, viabilidad, cultivo, y se envió una muestra al laboratorio de investigación del Hospital Nacional de Niños, para determinar por medio de citometría de flujo, la cantidad de células CD34+ recolectadas.

Las variables analizadas en el estudio son: volumen sanguíneo procesado, volemias procesadas, recuento de leucocitos prerrecolección, recuento de leucocitos en la recolección, recuento de células mononucleares en la recolección, recuento de CD34+ por recolección, recuento de células mononucleares/ $\mathrm{kg}$ del paciente por recolección y por cosecha (la suma de todas las recolecciones realizadas) y $\mathrm{CD} 34^{+} / \mathrm{Kg}$ del paciente por recolección y por cosecha.

Para evaluar la recuperación postrasplante, se analizan tiempo de recuperación de leucocitos en días y tiempo de recuperación en días de las plaquetas por $\mu$ l. Los parámetros utilizados para definir la recuperación son: un conteo mayor o igual a 500 leucocitos/ $\mu$ l en sangre periférica y un recuento de plaquetas mayor o igual a 20000 plaquetas/ $\mu 1$, luego de transcurrido un periodo no menor de 48 horas desde la última transfusión de plaquetas. ${ }^{5,13}$

En general, se realizaron 2 recolecciones por paciente, en días consecutivos; el objetivo en los procedimientos era obtener al menos recuentos $\geq$ a $2 \times 10^{6}$ células CD $34^{+}$por kilogramo de peso del paciente, por cosecha.

El análisis de datos se realiza con el programa estadístico SPSS 19.0, aplicando modelos de regresión, pruebas de $t$ Student, para 2 muestras y estadística descriptiva.

\section{Resultados}

En el periodo analizado de 2 años se realizaron 52 procedimientos de aféresis a 26 pacientes; los datos fueron analizados por recolección individual y por cosecha total obtenida de cada paciente; los diagnósticos se distribuyen en 3 grupos: mieloma múltiple (27\%), linfoma no Hodgkin (27\%) y linfoma Hodgkin (46\%).

En el estudio se analizaron 2 tipos de variables: unas son parámetros hematológicos propios del paciente y otras propias del procedimiento, con el fin de establecer cuáles de estas variables pueden ser utilizadas como parámetros que permitan predecir una buena recolección. El resumen de los datos obtenidos por aféresis se presenta en el Cuadro 1.

El promedio de leucocitos en la población estudiada antes de la recolección (postestimulación) fue de 30706/ $\mu 1$, y cada

Cuadro 1. Resumen de los parámetros analizados por aféresis $(n=26)$. Banco de Sangre, Hospital México. 2010-2012

\begin{tabular}{|c|c|c|c|}
\hline & Mínimo & Máximo & Media \\
\hline $\begin{array}{l}\text { Leucocitos } \times 10^{3} \\
\text { postestimulación }\end{array}$ & 3760,00 & 85420,00 & 30706,53 \\
\hline $\begin{array}{l}\text { Leucocitos } \times 10^{3} \\
\text { recolectados }\end{array}$ & 55,02 & 457,89 & 215,22 \\
\hline Mononuclearesx100 & 22,10 & 100,00 & 62,30 \\
\hline CD34x100 & 0,02 & 1,20 & 0,19 \\
\hline CD34 absoluto & 44,00 & 2464,00 & 457,15 \\
\hline $\begin{array}{l}\text { Volumen } \\
\text { recolectado }\end{array}$ & 120,00 & 255,00 & 235,94 \\
\hline Peso & 48,00 & 98,00 & 72,23 \\
\hline $\begin{array}{l}\text { Mononucleares/kg } \\
\times 10^{8}\end{array}$ & 1,00 & 8,64 & 4,24 \\
\hline Volumen procesado & 6920,00 & 20039,00 & 17972,55 \\
\hline Tiempo & 140,00 & 285,00 & 250,71 \\
\hline Volemia & 2945,00 & 5984,00 & 4586,30 \\
\hline CD34/Kg x10 & 0,13 & 8,32 & 1,53 \\
\hline
\end{tabular}




\begin{tabular}{|c|c|c|c|}
\hline \multicolumn{4}{|c|}{$\begin{array}{l}\text { Cuadro 2. Resumen de parámetros analizados por } \\
\text { cosecha. Banco de Sangre, Hospital México. } \\
\text { 2010-2012 }\end{array}$} \\
\hline & Mínimo & Máximo & Media \\
\hline $\begin{array}{l}\text { Volemias } \\
\text { procesadas }\end{array}$ & 4,24 & 11,58 & 8,04 \\
\hline $\begin{array}{l}\text { Volumen } \\
\text { procesado (ml) }\end{array}$ & 18174,00 & 40049,00 & 35945,08 \\
\hline $\begin{array}{l}\text { Cantidad de } \\
\text { aféresis }\end{array}$ & 2,00 & 2,00 & 2,00 \\
\hline $\begin{array}{l}\text { CD34total/ } \mathrm{Kg} \\
\times 10^{6}\end{array}$ & 0,32 & 9,90 & 3,06 \\
\hline $\begin{array}{l}\text { MononuclearesI } \\
\mathrm{Kg} \times 10^{8}\end{array}$ & 2,50 & 13,78 & 8,48 \\
\hline
\end{tabular}

procedimiento duró en promedio 250,7 minutos, durante los cuales se procesaron aproximadamente $17972 \mathrm{ml}$ por paciente, lo que permitió recolectar alrededor de $235,9 \mathrm{ml}$ en cada aféresis, y obtener en este volumen, en promedio, 4,24 x10 células mononucleares/Kg de peso del paciente, entre las cuales $1,53 \times 10^{6}$ corresponden a células CD $34^{+}$(Cuadro 1 ).

Con un análisis estadístico de correlación bivariado, se encontró asociación significativa entre el $\mathrm{CD}_{3} 4^{+} / \mathrm{Kg}$ y la cantidad de leucocitos del paciente antes de la recolección (leucocitos post estimulación) p=0,028; además, se observó asociación entre el recuento de CD34 $/ \mathrm{kg}$ y los leucocitos $\times 10^{3}$ recolectados, y los mononucleares $/ \mathrm{kg} \times 10^{8}$ recolectados $(\mathrm{p}<$ $0,05)$. Las demás variables estudiadas no mostraron tener una relación directa sobre el rendimiento de las recolecciones de células CD34 positivas ( $p \geq 0,05)$.

De las 3 variables que correlacionan con el conteo de células $\mathrm{CD} 34^{+} / \mathrm{kg}$, únicamente el recuento de leucocitos postestimulación puede ser utilizado como un predictor de la eficacia de la recolección, ya que los otros 2 parámetros son parte del resultado final y solo sirven para estimar el conteo de CD34+ luego de la recolección.

\section{Rendimiento por cosecha total por paciente}

En promedio, a los pacientes se les procesaron 8,04 volemias de un mínimo de 3 programadas, para un promedio de 35945,1 mililitros de sangre procesada por paciente, con una duración total de la recolección de 501,4 minutos. Se logró recolectar una media de 8,48 x $10^{8}$ células mononucleares/Kg de peso, de las cuales, en promedio, $3,06 \times 10^{6} / \mathrm{kg}$ corresponden a células CD34 positivas.

\section{Rendimiento por cosecha, según diagnóstico}

En cuanto al rendimiento obtenido en las recolecciones según diagnóstico, cuyos datos se muestran en el Cuadro 3, se pueden observar leves diferencias en cuanto al rendimiento, pero estas no son significativas al comparar los grupos mediante una prueba de $t(p>0,05)$, por lo que el diagnóstico no influye en el rendimiento de las recolecciones, aunque se debe destacar que las poblaciones son pequeñas y de tamaños muy diferentes.

\section{Recuperación de parámetros hematológicos en los pacientes postrasplante}

Para evaluar en la práctica la eficacia del rendimiento obtenido en las recolecciones de células madre, se cuantificaron los tiempos de recuperación de parámetros hematológicos que permiten establecer si hay una implantación exitosa del injerto, como son los recuentos de plaquetas y de leucocitos postrasplante.

Del total de 26 pacientes a los que se les realizó recolecciones para autotrasplante, solo 22 fueron trasplantados, y 3 murieron antes de alcanzar los valores que sirven de criterio para evaluar recuperación, por lo que el porcentaje de mortalidad en este periodo fue del 13,66\%. Por tanto, el número de casos final para evaluar el tiempo de recuperación fue de 19.

En el Cuadro 4 se resume la información respecto a los días necesarios para recuperar los valores aceptables de leucocitos y plaquetas en ese periodo, según el rendimiento de las recolecciones.

El tiempo de recuperación de los leucocitos fue, en promedio, 15,05 días, y en todos los pacientes fue menor que el de recuperación de las plaquetas, el cual fue de 17,15 días en promedio. Si se establece el tiempo de recuperación general para estos pacientes, como el tiempo que se necesita para recuperar ambos conteos celulares en sangre periférica, este viene a ser el mismo de recuperación de plaquetas, ya que en todos los casos este parámetro fue el último en recuperarse y, en promedio, fue de 17,45 días, con un rango que va desde 11 hasta 25 días.

Al analizar si el recuento de células CD34+ infundidas influye en el tiempo de recuperación en alguno de estos parámetros evaluados, se obtiene una correlación directa entre el tiempo de recuperación de plaquetas y el conteo de $\mathrm{CD} 34+/ \mathrm{kg}$ de peso presentes en la recolección ( $p=0,043)$, lo que no se repitió en el caso de la recuperación de leucocitos ( $p>0,05)$.

Cuadro 3. Rendimiento de CD34/ kg x $10^{6}$ recolectadas, según diagnóstico. Banco de Sangre, Hospital México. 2010-2012

\begin{tabular}{|lccccc|}
\hline \multicolumn{1}{c}{ Diagnóstico } & Media & Máximo & Mínimo & N total & $\%$ \\
Linfoma de Hodgkin & 3,27 & 9,90 & 0,58 & 12 & 46,2 \\
Linfoma no Hodgkin & 2,70 & 6,29 & 0,32 & 7 & 26,9 \\
Mieloma múltiple & 3,05 & 5,28 & 0,78 & 7 & 26,9 \\
\hline
\end{tabular}




\begin{tabular}{|lccc|}
\hline $\begin{array}{c}\text { Cuadro 4. Días de recuperación postrasplante. Banco } \\
\text { de Sangre, Hospital México. 2010-2012 }\end{array}$ \\
\hline $\begin{array}{c}\text { Días de } \\
\text { recuperación }\end{array}$ & Mínimo & Máximo & Media \\
Leucocitos & 9,00 & 23,00 & 15,05 \\
Plaquetas & 11,00 & 25,00 & 17,15 \\
\hline
\end{tabular}

Otro parámetro, como el número de células mononucleares/ kg infundidas, no mostró tener una asociación significativa con los tiempos de recuperación de plaquetas ni de leucocitos ( $p$ $>0,05)$.

En general, no se evidenció efectos adversos durante las recolecciones en los pacientes (asociados al citrato, vasovagales, ni complicaciones por el catéter).

\section{Discusión}

Varios investigadores han abordado la influencia del régimen de medicamentos utilizado y la enfermedad primaria en la movilización de células CD34 positivas, mucho se ha estudiado en lo que se refiere a los equipos utilizados, el número de procedimientos necesarios por paciente y los parámetros que pueden emplearse para predecir una buena 0 mala recolecta.

Los parámetros utilizados para determinar el inicio de la recolección están dados por varios factores: 1) por calendario: entre el día 4 y el día 6;2) por un incremento mínimo de la cuenta total de leucocitos por arriba de $10000 / \mu \mathrm{L}$, idealmente $50000 / \mu \mathrm{L}$, y 3) la cuenta de células CD 34 positivas cercana a 20., ${ }^{1,414}$ En el estudio se cumplen estos parámetros, exceptuando la determinación de las células $\mathrm{CD} 34^{+}$en sangre periférica antes del procedimiento, ya que hasta ese momento no está disponible. Otros parámetros que pueden afectar el rendimiento en las recolecciones de células madre de sangre periférica, van desde los métodos para la movilización de las células madre, hasta el equipo por utilizar y el protocolo de la recolección. Dentro de este último se incluye otra serie de factores de importancia en el desempeño por obtener. Muchos autores difieren en cuanto a los protocolos adecuados y a los rendimientos mínimos necesarios en estos procedimientos, pero en lo que la mayoría está de acuerdo es en que cada centro médico debe establecer sus propios protocolos, con los parámetros que les ofrezcan el mejor rendimiento.

Existen beneficios de los trasplantes con células madre obtenidas de sangre periférica con respecto a las conseguidas directamente de médula ósea, entre los que destacan: menor riesgo de contaminación tumoral, tiempos de recuperación menores postrasplante, menor estadía hospitalaria y, por ende, menor costo económico., ${ }^{2,7}$ Sin embargo, la dosis adecuada de células madre necesarias para minimizar el tiempo entre la infusión y la recuperación de los parámetros celulares en sangre periférica, permanecen aún sujeta a debate.
En el estudio el rendimiento obtenido con este protocolo es de $3,06 \times 10^{6} \mathrm{CD} 34+/ \mathrm{kg}$ por cosecha, en promedio, valor que se encuentra entre los parámetros establecidos por la mayoría de centros médicos a nivel internacional, pero cuya utilidad en la práctica solo se puede observar al evaluar la recuperación postrasplante de los pacientes infundidos con estas células. Además, entre las variables analizadas en el estudio, únicamente el recuento de leucocitos prerecolección mostró ser un buen indicador para una recolección exitosa $(\mathrm{P}=0,028)$. Estudios anteriores hacen una correlación similar entre la dosis final de CD $34^{+} / \mathrm{kg}$ y el recuento de leucocitos prerecolección, pero esta se invierte cuando los recuentos de leucocitos son superiores a 35000 células por $\mu 1$, postulando que un exceso de leucocitos puede crear una banda de separación de células mononucleares que sobrepasa el diámetro del canal de recolección, incrementando el escape de células mononucleares y, por ende, de CD 34+, afectando la eficiencia de la cosecha; ;,14 aunque tal situación no se presentó en el estudio, se debe tener en cuenta que, en promedio, los recuentos de leucocitos prerecolecta fueron de $30706 / \mu \mathrm{L}$.

El otro parámetro evaluado para determinar el éxito del los procedimientos realizados en las recolecciones, es el tiempo de recuperación en los pacientes trasplantados con las células recolectadas. Figuerres y cols., ${ }^{13}$ en un estudio con pacientes pediátricos, no hallaron correlación entre los recuentos de CD34+/kg infundidos o células mononucleares infundidas, y el tiempo de recuperación de leucocitos y plaquetas, a diferencia de Weaver y cols., ${ }^{15}$ cuya correlación entre el número de CD34+/kg infundidas y el tiempo de recuperación en los niveles de plaquetas y leucocitos, sí fue significativa. En el estudio el parámetro que tardó más en recuperarse fue el de las plaquetas, con un promedio de 17,15 días, y por ende, este es el promedio de recuperación final en días de los pacientes, y aunque no se demuestra una correlación entre el recuento de células $\mathrm{CD} 34+/ \mathrm{kg}$ infundidas y el tiempo de recuperación de los leucocitos $(p>0,05)$, sí se encontró una correlación significativa entre este recuento y el tiempo de recuperación de las plaquetas $(\mathrm{p}=0,043)$.

Con los datos obtenidos se puede afirmar que la recolección de células madre de sangre periférica mediante la aféresis de grandes volúmenes, es segura y eficaz cuando se realiza dentro de los parámetros evaluados. Además, es evidente que para predecir una buena recolecta, según los datos obtenidos, solo el recuento de leucocitos prerecolección resulta ser de utilidad, ya que hasta el momento del estudio no se disponía de la posibilidad de cuantificar las células $\mathrm{CD} 34^{+}$prerecolección, dato que se incorporará pronto en el protocolo.

La recuperación hematológica evaluada mostró ser similar a la descrita en otras publicaciones, por lo que el procedimiento de recolección sumado al procedimiento médico postrasplante resultan satisfactorios, pero el único parámetro obtenido en la recolección que permite establecer un buen pronóstico en la recuperación del trasplantado, es la dosis de células CD34+/kg infundidas, aclarando que en este caso, no se toma en cuenta el estado físico del paciente, la etapa de la enfermedad, ni el tratamiento en el momento del trasplante. 


\section{Referencias}

1. Cooling L, Hoffmann S, Herrst M, Muck C, Armelagos H, Davenport R. A prospective randomized trial of two popular mononuclear cell collection sets for autologous peripherical blood stem cell collection in multiple myeloma. Transfusion 2010; 50: 100-119.

2. Aguilar D, Lordmendez D. Recolección de células progenitoras en niños. Rev Mex Med Tran, 2010; 3, Supl. 1: S71-S74.

3. Tempescul A, Ianotto J.C, Hardy E, Quivoron F, Petrov L, Berthou C. Peripheral blood stem cell collection in elderly patients. Ann Hematol 2010; 89: 317-321.

4. Akhtar S, Weshi A, Rahal M, Khafaga Y, Tbakhi a, Humaidan H, maghfoor I. Factors affecting autologous peripheral blood stem cell collection in patients with relapsed or refractory diffuse large cell lymphoma and Hodking lymphoma: a single institution results of 168 patients. Leuk Lymphoma 2008; 49:769-778.

5. Lopez F. Recolección de células progenitoras hematopoyéticas mediante aféresis de grandes volúmenes. Hemasferio 2012: 22-26.

6. Moreb J.S, Salmasinia D, Hsu J, Hou W, Cline C, Rosenau E. Long-TermOutcome after Autologous StemCell Transplantation with Adequate Peripheral Blood Stem Cell Mobilization Using Plerixafor and G-CSF in PoorMobilizer Lymphoma and Myeloma Patients. Adv hematol 2011, Article ID 517561, 8 pages doi:10.1155/2011/517561.

7. Wang S, Nademanee A, Oian D, Dagis A, Park H-S, Fridey J, et al. Peripheral blood hematopoietic stem cell mobilization and collection efficacy is not an independent prognostic factor for autologous stem cell transplantation. Transfusion 2007; 47: 2207-2216.

8. Hoggatt J, Pelus L.M. Mobilization of hematopoietic stem cells from the bone marrow niche to the blood compartment. Stem Cell Res Ther 2011: 2-13.
9. Awan F.T, Kochuparambil S.T , DeRemer D, Cumpston A, Craig M, Jillella A, Hamadani M. Plerixafor Salvage Is Safe and Effective in Hard-to-Mobilize Patients Undergoing Chemotherapy and Filgrastim-Based Peripheral Blood Progenitor Cell Mobilization. J Oncol 2012, Article ID 931071, 5 pages doi:10.1155/2012/931071.

10. Vantelon J.M, Koscielny S. Brault P, Bourhis J.H, Ribrag V, Pico $\mathrm{J}$, et al. Scoring system for the prediction of successful peripheral blood stem cell (PBSC) collection in non.Hodgkin's lymphoma (NHL): application in clinical practice. Bone Marrow_Transplant 2000; 25: 495-499.

11. Subair A. C, Grant r, Wu W, Tun H, Rivera C, Moreno-Aspitia A, et al. Platelet count is a sensitive predictor of autologous peripheral blood progenitor cell collection yield in previously treated plasma cell disease patients. Transfusion 2008; 48: 1106-1114.

12. Ford C.D, Chan K.J, Reilly W.F, Petersen F.B. An evaluation of predictive factors for CD34+ cell harvest yields from patients mobilized with chemotherapy and growth factors. Transfusion 2003; 43: 622-625.

13. Figuerres E, Haut P.R, Olzewski M, Kletzel M. Analysis of parameters affecting engraftment in children undergoing autologous peripherical blood stem cell transplants. Bone Marrow Transplant 2000; 24: 583-588.

14. Kim J.e, Yoo C, Kim S, Lee D.H, Kim S, Lee J.S, Suh C. Optimal timing of G-CSF administration for effective autologous stem cell collection. Bone Marrow Trasplant 2011; 46: 806-812.

15. Weaver C.H, Hazelton B, Birch R, Palmer P, Allen C, Schwartzberg L, West W. An analysis of engraftment kinetics as a function of the CD34 content of peripheral blood progenitor cell collections in 692 patients after the administration of myeloablative chemotherapy. Blood 1995; 86: 3961-3969. 